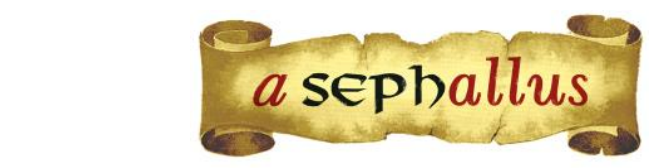

Revista aSEPHallus de Orientação Lacaniana

Núcleo Sephora de Pesquisa sobre o Moderno e o Contemporâneo

ISSN 1809 - 709 X

\title{
De la structure autistique ${ }^{1}$
}

Jean-Claude Maleval

Psychanalyste. Membre de l'École de la cause freudienne et de l'Association Mondiale de Psychanalyse (Paris, France). Professeur émérite de psychologie clinique à l'Université de Rennes 2 (Paris, France)

E-mail: jean-claude.maleval@univ-rennes2.fr

Resumé : La notion de structure subjective autistique a été introduite par Rosine et Robert Lefort dans les années 1990 et caractérisée par l'absence d'aliénation significative, de lalanguage, de S1 et d'objet a. Ces indications sont encore en discussion de nos jours car, pour comprendre la complexité de la clinique du spectre autistique, ces auteurs n'ont eu l'idée que de diviser le sujet en réel du double. L'extraction plus précise de la structure autistique nécessite des étapes supplémentaires à partir d'éléments qu'ils ne possédaient pas: nouveaux documents cliniques, études sur la cognition de l'autiste et formation du concept de bord. De nos jours, il semble possible d'appréhender la structure autistique à partir d'éléments plus vastes, tels que: une rétention initiale des objets pulsionnels; une aliénation conservée, qui fonctionne sans la charnière du maître-signifiant; un appareil de jouissance par le bord.

Mots-clés: psychanalyse; l'autisme; la structure; autiste de haut niveau; Asperger; signe.

Da estrutura autista: A noção de estrutura subjetiva autística foi introduzida por Rosine e Robert Lefort nos anos 1990 e caracterizada pela ausência de alienação significante, de lalíngua, de S1 e de objeto a. Estas indicações são ainda objeto de debate atualmente pois, para apreender a complexidade da clínica do espectro autista, estes autores só dispunham da noção de divisão do sujeito no real do duplo. A extração mais precisa da estrutura autística requer alguns passos suplementares a partir de elementos que eles não dispunham: novos documentos clínicos, estudos sobre a cognição dos autistas e a formação do conceito de borda. Hoje em dia parece possível apreender a estrutura autista a partir de características maiores, tais como: uma retenção inicial dos objetos pulsionais; uma alienação retida, que se opera sem a dobradiça do significantemestre; uma aparelhagem do gozo pela borda.

Palavras-chave: psicanálise; autismo; estrutura; autista de alto nível; Asperger; signo.

On autistic structure: The notion of autistic subjective structure was introduced by Rosine and Robert Lefort in the 1990s and characterized by the absence of significant alienation, from lalangue, from S1 and from object a. These indications are still under discussion today because, in order to understand the complexity of the autistic spectrum clinic, these authors only had the notion of dividing the subject into the real of the double. The more precise extraction of the autistic structure requires some additional steps from elements that they did not have: new clinical documents, studies on the cognition of the autistic and the formation of the concept of edge. Nowadays it seems possible to apprehend the autistic structure from larger features, such as: an initial retention of the pulsional objects; a retained alienation, which operates without the hinge of the master-signifier; an apparatus of enjoyment by the edge.

Keywords: psychoanalysis; autism; structure; high-level autistic; Asperger; sign. 


\section{De la structure autistique}

Jean-Claude Maleval

Il paraît aujourd'hui possible d'appréhender la structure autistique à partir de trois caractéristiques majeures :

- Une rétention initiale des objets pulsionnels

- Une aliénation retenue, qui s'opère sans la cheville du signifiant-maître.

- Un appareillage de la jouissance par le bord.

L'hypothèse de l'existence d'une structure autistique conforte la notion de spectre de l'autisme introduite en 2013 par le DSM-5 pour se substituer aux "Troubles envahissants du développement". Est-il fondé de postuler des possibilités de passage des formes les plus sévères de l'autisme kannérien à la socialisation des autistes de haut niveau ? Le devenir de Donald Gay Tripplet, le cas $\mathrm{n}^{\circ} 1$ de l'article princeps de Kanner, est le plus démonstratif et le moins contestable de ces évolutions possibles. Observé dans les années 1930 au Johns Hopkins Hospital de Baltimore, il profitait en 2010 d'une paisible retraite dans le Mississipi. Après avoir travaillé comme caissier dans la banque de ses parents, il vivait indépendant et seul, conduisait toujours sa voiture et continuait à cultiver ses loisirs : le golf et les voyages (Dovan \& Zucker, 2010). À trois ans, Grandin était une enfant muette, colérique, fixée sur les objets tournants, cherchant à être seule, et jouant avec ses déjections. Elle considère, comme beaucoup de spécialistes, que la sévérité des symptômes vers l'âge de deux ou trois ans est souvent sans rapport avec le pronostic (Grandin, 1997).

\section{Une rétention initiale des objets pulsionnels}

Il fait aujourd'hui quasiment consensus que les deux signes les plus précoces de l'autisme sont repérables très tôt, le premier, vers trois mois, la fuite du regard, le second vers neuf mois, le manque d'attention conjointe. Ce dernier signifie que lorsque l'enfant pointe un objet du doigt, il n'utilise pas son regard pour attirer l'attention de l'adulte vers la cible d'intérêt. Ces deux signes sont du même ordre : I'enfant autiste n'utilise pas son regard pour communiquer. Le phénomène n'est pas anecdotique : il persiste encore chez la plupart des autistes de haut niveau.

Or, il n'y a pas que les interactions scopiques qui sont évitées par le petit autiste : ce sont tous les objets qui sont mobilisés dans les premiers échanges avec les parents qui sont plus ou moins refusés ou retenus, à savoir la voix, les selles et la nourriture.

Kanner note d'emblée la fréquence d'un refus précoce des aliments. La rétention de l'objet anal n'est pas moins fréquente que celle du regard et de la voix. Le recours aux lavements est parfois nécessaire pour éviter l'occlusion intestinale. D'autre part, une proportion importante d'autistes restent muets lors de leurs premières années, refusant ainsi de céder leur voix aux 
attentes de l'Autre. Certains souffrent de ce refus qui dépasse leur volonté ; d'autres semblent se retenir par prudence. "Pourquoi ne parlons-nous pas normalement? se demande Higashida sur son ordinateur. Je ne sais pas. Ce n'est pas que nous ne voulons pas, c'est que nous ne pouvons pas, et nous en souffrons" (Higashida, 2014, p.46).

Il arrive à Sellin de prononcer quelques mots, mais il n'est pas en mesure de préciser ce qui détermine son mutisme :

...parfois je peux dire une chose à l'improviste

je suis alors très étonné moi-même de ce qui a pu se

produire je n'ai pas d'explication

je pense simplement que c'est une preuve que la

faculté de parler existe

reste à trouver un moyen de sortir du mutisme (Sellin, 1998, p.25).

Certains autistes peuvent témoigner que lâcher la voix est vécu comme une angoissante perte de substance qui équivaudrait à une mutilation : "Comme nous le diront par la suite plusieurs enfants antérieurement mutiques [rapporte Bettelheim], ils ne parlaient pas parce que cela aurait vidé leur cerveau" (Bettelheim, 1969, p.89).

Pourquoi la rétention du regard? Parce que, dit l'un, c'est trop inquiétant. Pourquoi chercher à éviter la défécation ? Par peur, confie un autre, que les poumons explosent. Pourquoi le mutisme ? Par crainte, pour certains, de se vider le cerveau.

Tous ces phénomènes suggèrent fortement que l'autisme s'enracine très tôt dans une crainte d'entrer en interaction avec les autres, qui ne relève pas d'une incompréhension des relations sociales, mais d'une angoisse irrationnelle que le sujet ne maîtrise pas. L'enfant autiste refuse initialement de faire entrer les objets pulsionnels dans l'échange ; c'est ce que discerne Donna Williams, en soulignant que dans son enfance tout ce qui tournait "autour de l'acte de donner et de recevoir lui restait totalement étranger" (Williams, 1992, p.66).

À la source de l'autisme se trouve une rétention des objets pulsionnels, qui suscite un trouble de la communication, car leur cession est au fondement de l'entrée dans la relation avec I'Autre. Les premiers cris de l'autiste sont monocordes, sans modulation, chez des bébés étonnamment calmes, ou bien ce sont de fréquents hurlements que rien n'arrête. Dans les deux cas, les parents ne sont pas mis en position de les interpréter comme des demandes.

\section{Une aliénation sans la cheville du signifiant-maître}

Le refus de faire entrer la voix dans l'échange génère deux conduites fréquentes chez l'autiste : le mutisme quand on l'incite à répondre et se boucher les oreilles quand on lui parle. Comment acquérir le langage dans ces conditions ? Il semble que l'autiste recourt à deux manières bien différentes qui possèdent en commun d'éviter le don de la voix. Mottron met l'accent sur ce 
point: "les enfants autistes [constate-t-il], apprennent le langage sans passer par deux de ses composantes les plus importantes chez la personne typique, soit l'interaction sociale et la signification" (Mottron, 2016, p.188). Il existe deux canaux majeurs d'entrée dans le langage pour l'autiste, l'un passe par un babil pauvre et par l'écholalie, l'autre principalement par l'écrit. Dans les deux cas, un évitement de l'interaction sociale est recherché. La première entrée ne prend pas en compte la signification; la seconde $n^{\prime}$ implique pas la jouissance vocale. Il en résulte une scission frappante entre deux modes d'expression.

Souvent, quand ils parlent, constate un clinicien, les autistes le font d'une voix atone, mécanique, comme si [...] la part musicale de la langue était dissociée du sens, comme s'ils avaient le choix entre parler sans musique ou faire des sons sans sens : sens brut ou son brut, code informatif ou émotion sensitive, mais jamais les deux articulés (Hébert, 2006, p.208).

Le son brut, issu du babil et des écholalies, porteur d'émotions sensitives, non communicables, se déploie en une langue verbeuse, propre à des satisfactions vocales solitaires ; tandis que l'acquisition du langage par l'écrit favorise l'émergence d'un "sens brut", d'un "code informatif ", d'une langue factuelle apte à la communication, mais coupée des affects.

\section{Le babil autistique}

Dès les années 1970, les psychologues ont constaté que des conduites d'évitement sont rencontrées très tôt chez le bébé à devenir autistique, une fuite du regard et une absence de pointage proto-déclaratif apparaissent fréquents dès la première année. On s'accorde à considérer que ces bébés ne recherchent pas la réciprocité qui "s'organise autour de l'échange de regards, de vocalisations, de tour de rôles entre la mère et l'enfant" (Richer, 1978, p.69). Pendant les premiers mois, certains enfants autistes ne babillent pas, mais pleurent ou hurlent quand ils sont éveillés. D'autres restent silencieux. Quand le babil existe, il apparaît le plus souvent monotone (comparable à celui d'un bébé tombant de sommeil), sans entrain, sans inflexion intentionnelle. De surcroît, il ne s'adapte pas aux productions maternelles. Certains autistes babillent dans la solitude, mais s'interrompent en présence d'un parent.

Les plus récentes études sur le babil et les vocalisations du bébé autiste précisent que leurs caractéristiques majeures résident dans une pauvreté statistiquement significative et dans une moindre recherche des échanges interactifs. De zéro à six mois, la fréquence des vocalisations entre les bébés à devenir autistique et les autres s'avère équivalente ; en revanche à partir de six mois, période d'apparition du babil, la différence apparaît nette (Chericoni et al, 2016). Quand le babil ne fait pas défaut, les études constatent son atypicité : outre sa moindre fréquence, il est peu coordonné avec le regard, il est volontiers solitaire plus qu'interactif, bref il apparaît moins 
socialement orienté.

Ce point est essentiel puisque les éléments du babil ne peuvent acquérir une signification qu'à partir de la réponse de l'Autre. L'entrée dans le langage, souligne Lacan, s'opère à la faveur de la mutation du cri en appel. Pour que le sujet devienne invoquant, une cession de l'objet voix est nécessaire, il faut que le sujet accepte de faire "résonner son cri" dans le vide de l'Autre, ce qui rend possible de "retrouver les marques de réponses qui furent puissantes à faire de son cri un appel". Quand Lacan écrit ces lignes en 1960, il n'a pas encore dégagé la notion de S1, mais il désigne déjà comme des "insignes les marques où s'inscrivent le tout-pouvoir de la réponse" (Lacan, 1966, p.679). Il indique ainsi que l'entrée dans le langage se fait pour l'infans en prélevant, parmi les signifiants proposés comme réponse à son cri, l'un d'entre eux, qui devient un S1 propre à convertir le cri en appel. S'il en est bien ainsi, si le signifiant de la réponse de l'Autre doit être préalablement entendu pour que le cri s'efface sous un S1 d'appel, alors l'aliénation dans le discours de l'Autre devrait être décelable dès le babil. Or, c'est bien ce que soutiennent maintenant les linguistes contemporains qui ne le réduisent plus à des vocalisations insensées. Ils constatent qu'il s'agit d'un proto-langage qui préside à l'entrée dans le langage. Selon eux, le babillage n'est pas le langage, mais il est un langage qui fournit un cadre pour le développement de la parole (Boysson-Bardies, 1996, p.60), si bien qu'il n'y a pas de discontinuité entre les formes du babillage et celle des premiers mots : "certains enfants donnent ainsi l'impression de choisir leurs premiers mots parmi les sons du babillage qu'ils ont aimé produire" (Lefort \& Lefort, Paris, 2003, p.166). Il est cohérent à cet égard que la pauvreté du babil de l'autiste se prolonge entre douze et dix-huit mois par des premiers mots moins nombreux et moins adressés (Chericoni et al, 2016, p.8). On a longtemps cru que le babil n'était qu'un chaos non structuré ; or il s'est avéré que dès le huitième mois, il révèle une précoce adaptation aux principes structuraux de la langue maternelle. Le babil d'un enfant anglais est différenciable de celui d'un français, d'un suédois, d'un algérien ou d'un japonais, de sorte qu'il témoigne déjà d'un ancrage du sujet et de son énonciation dans le discours de l'Autre.

On a pu montrer que l'enfant typique "accompagne de vocalisations particulières des demandes, des manipulations d'objets comme le rangement des cubes, ou des gestes comme celui de s'asseoir " (Blake \& Boysson-Bardies, 1992); si bien que le babil peut servir à exprimer désirs, intérêts et refus. Il n'est pas "autistique" au sens de "hors-communication", c'est si vrai que l'adulte peut I'utiliser afin de provoquer des réponses de la part de bébés. Les Lefort font I'hypothèse d'une absence de babil chez l'enfant autiste afin de soutenir qu'il ne fait pas son entrée dans le langage en passant par "le babil de lalangue" (Lefort \& Lefort, 1998, p.315) de telle sorte qu'il n'y aurait pour lui ni S1, ni lalangue. Or, les études les plus récentes mettent en évidence que la caractéristique majeure du babil autistique n'est pas son absence, mais sa "pauvreté statistiquement significative" et son peu d'orientation sociale ; ce qui induirait plutôt l'hypothèse d'une lalangue "pauvre". 
Le rapprochement entre le babil et la lalangue est opéré par Lacan lui-même puisqu'il précise avoir choisi le terme de lalangue parce qu'il a voulu le faire aussi proche que possible du mot lallation (Lacan, 1974). Or, celui-ci est synonyme de "jasis", "gazouillis" ou "babillage", ces termes désignent une étape de développement du langage, qui succède vers six mois aux premières vocalisations et qui décline progressivement à partir de douze mois avec l'apparition des premiers mots. La lalangue, cette pure batterie signifiante, sans grammaire, constituée de S1, fondée dans les homophonies infantiles, prend ses racines dans le babil. Elle constitue ce par quoi s'opère une incorporation du signifiant : "Lalangue" affirme Lacan, "c'est au regard de la jouissance phallique un brin de jouissance. Et c'est en ça que ça étend ses racines si loin dans le corps " (Lacan, 1974). Dès lors, c'est par son entremise que se produit l'animation de la jouissance du corps; "tandis qu'elle est solidaire de la réalité des sentiments qu'elle signifie" (Lacan, 1974). "Il y a une rencontre entre lalangue et le corps", précise J-A Miller, "et, de cette rencontre, naissent des marques qui sont des marques sur le corps. Lacan appelle sinthome la consistance de ces marques. C'est en quoi il peut réduire le symptôme à être un événement de corps, quelque chose qui est arrivé au corps du fait de lalangue" (Miller, 2005, p.152). En outre, elle permet au sujet d'appréhender des modifications corporelles sous forme d'affects conscients : ces derniers, précise Lacan, sont ce qui résulte de la présence de lalangue en tant que, de savoir, elle articule des choses qui vont beaucoup plus loin que ce que l'être parlant supporte de savoir énoncé " (Lacan, 1975b, p.127).

En raison de l'absence de transformation du cri en appel, l'autiste reçoit les signifiants de manière passive et non interactive, de sorte qu'ils n'ont pas la capacité à faire sens en s'articulant à d'autres signifiants. La spécificité de la lalangue autistique réside en ce que d'elle n'émergent pas des signifiants-maîtres. Elle ne se prête pas aux articulations qui produisent l'animation de la jouissance du corps. Il convient alors de préciser, comme le notent parfois les Lefort, que ce n'est pas le S1 tout seul qui fait défaut dans l'autisme, mais que "la cheville du signifiant-maître initial, le S1, est forclose" (Lefort \& Lefort, 1998, p.26). Au premier plan de la clinique de l'autisme infantile, par l'entremise de la langue verbeuse et des vocalisations involontaires, vient une lalangue qui ne s'efface pas, faute de s'articuler au discours de l'Autre. Elle est constituée de SI tout seuls, juxtaposés. Ils sont au service, non pas de la communication, mais de satisfactions solitaires. La pauvreté de la lalangue autistique réside en ce que ses S1 n'ont pas la potentialité de devenir des signifiants-maîtres propres à marquer le corps d'une lettre au principe d'un sinthome.

\section{Les vocalisations involontaires.}

En 1946, à la suite d'observations de vingt-trois enfants autistes, Kanner constata un phénomène étrange concernant le "mutisme" de huit d'entre eux: "en de rares occasions [il] est interrompu par l'émission d'une phrase intégrale dans des situations d'urgence" (Kanner, 2002).

Que disent-ils le plus souvent dans ces moments-là ? La première phrase prononcée par 
Birger Sellin est : "rends-moi ma boule" adressée à son père qui venait de lui prendre l'un de ses objets autistiques (Sellin, 1994, p.24). Un garçon de cinq ans, rapporte Berquez, "que personne n'avait jamais entendu prononcer un seul mot de sa vie, s'est trouvé gêné quand la peau d'une prune s'est collée à son palais. Il s'exclama alors distinctement: "Enlevez-moi ça", puis il retomba dans son mutisme antérieur. Un autre enfant mutique de quatre ans se faisant examiner par un pédiatre cria: "Je veux rentrer" et, un an plus tard, à l'occasion d'une hospitalisation pour une bronchite, il s'écria: "Je veux retourner" (Berquez, 1983, p.107).

Toutes ces phrases possèdent un point commun : la présence du sujet de l'énonciation s'y trouve nettement marquée. La vocalisation involontaire n'est pas une laborieuse construction intellectuelle, mais une parole qui sort des tripes. Son caractère impératif témoigne de la jouissance vocale qui la mobilise. Or, tout particulièrement quand l'énoncé est adressé, l'expérience s'avère déchirante pour l'enfant autiste. Ce n'est guère qu'au comble de l'angoisse qu'il peut laisser échapper une telle vocalisation. Elle est vécue comme une mutilation, car mettant en jeu, non seulement l'altérité, mais une cession de l'objet de la jouissance vocale. Bien loin de réitérer cette expérience angoissante, le sujet cherche à se protéger de son renouvellement, en se murant dans un silence encore plus profond.

L'énonciation inattendue ne surgit que dans des situations inquiétantes, présentant un caractère de nécessité ou d'urgence. Dans ces circonstances, elle n'est pas un choix subjectif réfléchi et assumé, mais une sorte de réflexe suscité par le contexte. Le sujet ne peut moduler ce qu'il vient de dire, ni se paraphraser, ni commenter, ni effectuer un retour réflexif. Ces phénomènes témoignent, selon Danon-Boileau, psychanalyste et linguiste, d'une "parole automatique" dans laquelle la pensée ne sert pas de médiation, puisque la situation mobilise directement l'affect ou la motricité qui fait sortir les mots par la bouche (Danon-Boileau, 2002, p.213). Il la rapproche de jurons ou d'exclamations de surprise qui échappent au locuteur. Les vocalisations involontaires ne représentent pas le sujet: elles constituent une extraction de la jouissance vocale équivalente à une défécation sans ressenti, à une alimentation sans faim, ou à un regard lâché dans le vide. Elles sont constituées de S1 tout seuls.

\section{La langue verbeuse.}

En 1975, à une époque où la représentation la plus répandue des autistes en fait encore des êtres muets, Lacan surprend en indiquant qu'il les trouve "plutôt verbeux": Que vous ayez de la peine à entendre, à donner sa portée à ce qu'ils disent, constate-t-il, n'empêche pas que ce sont des personnages plutôt verbeux " (Lacan, 1985, p.20). Il met ainsi l'accent sur un pan essentiel de leur clinique: celui où vient au premier plan une langue de $\mathrm{S} 1$ tout seuls. Les vocalisations involontaires et les soliloques de la langue verbeuse sont faites du même matériau. Les unes et les autres sont mobilisées pour des satisfactions solitaires et non pour initier un échange verbal. C'est ce que constatait Lacan à propos de Dick, pris en analyse par Mélanie Klein, quand il avait quatre 
ans : "cet enfant", disait-il, "n’a pas le désir de se faire comprendre, il ne cherche pas à communiquer, ses seules activités plus ou moins ludiques sont d'émettre des sons et de se complaire dans des sons sans signification, dans des bruits" (Lacan, 1975a, p.95).

La langue verbeuse trouve une source majeure dans l'écholalie : elle se nourrit des échos du double et non des réponses de l'Autre. "Le langage oral autistique, observe Mottron, quand il se met à apparaître est emprunté à des situations non interactives (chansons, publicité, prières, génériques) et non immédiatement référentielles" (Mottron, 2016, p.202). Quand les enfants autistes ne sont pas mutiques, leurs verbalisations originales suggèrent à Kanner les notions de "langage de perroquet" ou d' "écholalies à retardement". Parfois, les parents notent qu'ils acquièrent avec aisance des mots nouveaux, sans apprendre pour autant à parler, car leur parole n'est pas porteuse d'un message. Ils décrivent le phénomène en notant que l'enfant prononce des mots, mais ne les utilise pas.

L'enfant avançant en âge, la langue verbeuse prend souvent des formes qui se complexifient, elle peut sembler devenir adressée, mais elle reste fondamentalement un soliloque. Williams confie qu'elle était bonne en lecture à l'école, parce que c'était pour elle "l'occasion d'écouter avec délectation le son de [sa] propre voix d'une façon plus acceptable qu'à l'ordinaire " (Williams, 1992, p.49). En effet, elle dérangeait par ailleurs la classe en se parlant sans cesse à haute voix.

On disait, commente-t-elle, que j'aimais tout simplement le son de ma propre voix. C'était probablement juste. On me trouvait intelligente, peut-être, commente-t-elle, mais je n'étais guère sensée. Je parlais moins aux gens que je ne soliloquais par-dessus leur tête, comme si toute conversation devait se résumer à cela" (Williams, 1992, p.44, p.50).

Le verbiage autistique peut être mobilisé pour des usages divers, même pour des tentatives allusives de communication, mais, outre les satisfactions qu'il procure, ses fonctions majeures sont de protection: "un outil de dissimulation ou d'attaque", note Williams (Williams, 1992, p.140). Il contribue à garder la maîtrise du pseudo-échange et à maintenir l'autre à distance.

Il arrive qu'émerge de la langue verbeuse des suites réglées: "Thierry, quatre ans quatre mois, relate Lemay, récite ainsi sur un ton précieux le nom de tous ses camarades de prématernelle. José, quatre ans un mois, fait l'énumération des multiples rues qui entourent sa maison" (Lemay, 2004, p.146). De tels efforts pour discerner de l'ordre dans le langage et dans un monde chaotique peuvent conduire l'autiste à tenter de le construire lui-même en se forgeant une langue privée.

\section{Les langues privées.}

L'autiste n'aime pas les équivoques de la langue. Pour s'en prémunir, quand il cherche à 
communiquer, il s'attache à une langue factuelle qu'il voudrait aussi précise qu'un code, nous y insisterons ; mais il lui arrive aussi d'élaborer une langue à son propre usage. Il suppose qu'elle serait mieux appropriée à l'expression de ses émotions ou mieux à même de le conduire vers "la clarté". Annick Deshays fait la première hypothèse: "Un autiste raisonne très isolément", note-telle. "Il joue avec les mots tout en les codifiant selon son état émotionnel, il fabrique son dictionnaire " (Deshays, 2009, p.25). Sellin penche plutôt pour la seconde:

\author{
trouver magistralement, écrit-il, une langue simple essentielle \\ intelligible par tous articulation résonnant comme des \\ êtres de brume \\ les langues racontant avec sensibilité ce qui empêche \\ les êtres de brume d'atteindre la clarté de la masse \\ humaine gigantesque et sûre (Sellin, 1998, p.117).
}

Bien entendu, quand elle est ébauchée, le rêve qu'elle soit "intelligible par tous" s'évanouit rapidement. Au détour du témoignage sur sa vie intérieure, Sellin confie avoir plus ou moins esquissé l'élaboration d'une telle langue privée.

J'aimerais raconter aujourd'hui comment cela se passe dans le foyer trop 'tonukohass' je vais laisser ce mot il signifie ne t'énerve pas dans le langage sans complément c'est un langage pour tous les comme on dit couillons je l'ai inventé cette langue a également une grammaire personnelle 'riokeea' signifie donc les cheveux de la natte sont longs aujourd'hui (Sellin, 1994, p.86-87).

Cette langue privée, composée de néologismes, pourrait posséder un dictionnaire et une grammaire, mais ceux-ci n'étant connus que de leur inventeur, elle ne saurait délivrer à l'auditeur que des significations hermétiques.

Dès les premières vocalisations des enfants autistes apparaît parfois une tendance à créer une langue privée. Même quand elle comprise par les proches, elle n'est guère forgée pour servir à la communication : il s'agit plutôt d'une recherche de satisfactions verbales solitaires plus ou moins accompagnées d'une quête d'ordre et de clarté. Ainsi, vers trois ans et demi, Théo inventa une langue néologique que son entourage parvint à comprendre. Ses proches se mirent alors à lui parler en cette langue. Or, note sa mère, Valérie Gay, il refusa "que nous utilisions son langage en retour. Nous devions continuer de lui parler comme d'habitude et il nous répondait avec son langage réinventé" (Gay-Corajoud, 2018, p.95). Théo s'affirmait clairement en cette circonstance comme maître d'un langage qu'il cherchait à forger mais non à partager. Quand il eut quatre ans, son entourage et son orthophoniste entreprirent de noter sa langue privée. Ils en établirent un 
dictionnaire comportant cent cinquante mots ${ }^{2}$. Parmi ceux-ci, sept verbes et les vingt premiers chiffres, tandis que les substantifs sont largement dominants. Certains termes sont des déformations du discours de I'Autre (crocodile/Co-co-gli; Gâteau/Ako), mais beaucoup sont des inventions sans rapport avec celui-ci (Livre/yiyi; Chaussure/ya-ya; Lit/odo; Pommade/ba). Deux caractéristiques de cette langue privée mettent clairement en évidence qu'il ne s'agit pas d'une lalangue. Cette dernière selon Lacan est assujettie à l'équivoque (Lacan, 2001, p.490), elle ne se soutient que de malentendus et d'homophonies, de sorte qu'il est exclu de pouvoir la ranger dans un dictionnaire. D'autre part, J-A Miller considère comme caractéristique de la lalangue d'être une "multiplicité inconsistante" (Miller,1975, p.27), ce que n'est pas la langue privée de Théo, puisqu'elle est au moins partiellement organisée, en incluant la suite des nombres de 1 à $20^{3}$. Cette langue ne comporte aucun terme abstrait, le sens de chacun de ses éléments y est fixé, référant de manière non ambiguë à un objet précis. Elle cherche à mettre ordre et clarté dans les équivoques du langage. Il est remarquable que Théo n'accorde à personne d'autre le droit d'en faire usage : les satisfactions que lui procure cet objet de son invention lui sont réservées. Il n'entend pas les partager. Il est probable que si l'Autre venait à en user, il risquerait d'en corrompre la clarté.

La langue de Théo est conforme à ce que l'on observe chez des autistes d'Asperger plus âgés: elle fuit les équivoques et elle est en quête de suites réglées. Elle peut permettre une certaine communication avec l'entourage, mais elle n'est pas forgée pour cela. Si quelques parents parviennent à la comprendre, c'est essentiellement parce qu'ils se sont exercés à la déchiffrer, ce n'est pas à l'initiative de leur enfant.

Des langues privées plus complexes forgées par des autistes de haut niveau possèdent les mêmes caractéristiques : elles visent à réduire l'ambiguïté du langage et constituent des objets de satisfactions solitaires qui les rendent peu aptes à la communication. Il est des autistes qui relatent avoir éprouvé d'emblée une langue privée comme étant leur "langue maternelle" ${ }^{4}$. Parfois, celle-là n'est pas inventée, pour Tammet elle était déjà-là : dès sa petite enfance il adopta la langue des nombres. Il possède une perception synesthésique de chacun de ceux-ci, de sorte qu'ils lui apparaissent comme autant de formes, de couleurs, de textures et de mouvements. Le nombre 1 , par exemple, écrit-il, est d'un blanc brillant et éclatant, comme quelqu'un qui dirige le faisceau d'une lampe torche directement dans mes yeux" (Tammet, 2007, p.11). Chaque élément de sa langue maternelle est donc un objet complexe suscitant diverses perceptions. Les caractéristiques de ces dernières sont mises en avant pour le caractériser, de sorte que les nombres de Tammet peuvent être appréhendés au un par un, indépendamment de leur valeur différentielle; c'est pourquoi il est en mesure d'opérer des calculs en combinant leurs formes. Cette langue privée est chargée d'une jouissance exceptionnelle et lui procure d'intenses émotions. Quand il récita les 22500 décimales du nombre Pi qu'il avait mémorisé, il eut le sentiment, confia-t-il, d'avoir été "le porte-parole de Pi" (Tammet, 2018). La langue des nombres est un objet auquel il prête sa voix; 
ce n'est pas une langue subjectivée. Ce qu'il confirme quand il observe ne pas savoir d'où vient le sens qui s'attache à cette langue: il constate que Mercredi est un jour bleu, mais n'est pas en mesure de dire pourquoi. Elle lui donne certes le sentiment d'être en prise avec ses émotions, mais elle ne les exprime pas: elle les suscite. Il n'ignore pas qu'elle est impropre à les transmettre. Quand Tammet récite un poème de nombres qu'il a composé, son émotion est patente, mais elle reste obscure aux auditeurs. Sa langue de nombres n'est pas une lalangue, mais un objet qui procure des satisfactions solitaires, porteur d'un sens auquel le sujet consent, mais par lequel il ne peut communiquer son vécu. Il s'agit de surcroît d'un objet qui se prête à des manipulations remarquables lui permettant de faire de stupéfiantes mémorisations et d'étonnants calculs. Pour qu'elle se prête à ces derniers, il faut, bien entendu, qu'elle n'ait rien d'une multiplicité inconsistante et que ses éléments ne soient pas équivoques.

Avec son autre langue privée, le Mänti, Tammet cherche à parler "une langue bien à lui qui serait une expression tangible et communicable de [son] intimité" (Tammet, 2007, p.182). Cependant, tous les exemples donnés réfèrent à des mots qui désignent des objets ou des abstractions (Tammet, 2007, p.181) - aucun à des sentiments ou à des éprouvés corporels. Les termes du Mänti sont forgés de manière à avoir une certaine affinité avec les choses désignées. Le mot Mänti, choisi pour nommer cette langue, est construit d'après le finnois mänty, le pin, parce que "les pins croissent souvent en groupes nombreux et symbolisent l'amitié et la communauté" (Tammet, 2007, p.181). En outre, Tammet invente des mots qui, par un certain isomorphisme établissent des liens nouveaux entre les choses, par exemple hamma (dent) et hemme (fourmi un insecte qui mord). Le Mänti est souvent généré par un travail d'écriture opéré à partir de diverses langues étrangères. De par son "vocabulaire de plus de mille mots" (Tammet, 2007, p.181), il ne s'ancre pas dans les équivoques, de surcroît il possède une grammaire, deux caractéristiques qui le différencient nettement d'une lalangue. La prise en compte de données visuelles et concrètes semble majeure dans sa création, de sorte qu'il apparaît peu approprié à exprimer les données changeantes et subtiles des sentiments et des ressentis. En outre, il reste impropre à la communication: Tammet est le seul à comprendre le Mänti, langue que personne ne parle, langue sans Autre.

Le référent est sans équivoque en ce qui concerne les signes du Mänti ou ceux de la langue de Théo, tandis que les nombres de Tammet constituent "un vocabulaire numérique et visuel" qui est propre à son créateur (Tammet, 2007, p.13). Chaque terme de la langue privée est porteur d'un sens définissable. En revanche dans lalangue, les signifiants sont en attente d'un sens, qui n'advient que par une articulation au S2. Les éléments de la langue privée n'ont pas cette attente d'autres éléments qui viendrait les déterminer. Ils semblent se suffire à eux-mêmes.

La langue privée génère parfois des néologismes, mais ils sont d'une nature bien différente de ceux forgés par les paranoïaques. Pour ces derniers, ils possèdent un caractère d'évidence quant à leur signification qui les rend aptes à communiquer sans perte la chose même. Pour les 
autistes, ils s'insèrent souvent dans une langue secrète de leur création qui n'a pas pour fonction de servir à la communication. Les paranoïaques cherchent avec les néologismes à fonder une langue universelle; tandis que les autistes les dissimulent dans une langue privée.

Une des formes élaborées de la langue privée se construit avec les signifiants propres à composer un intérêt spécifique. Une intense jouissance s'attache à l'appropriation de ceux-ci et à la connaissance de leurs mises en ordre.

Je ne peux parler que pour moi, écrit Luke Jackson, un autiste Asperger, mais quand j'ai quelque chose en tête, alors le reste du monde cesse d'exister. Je suppose que l'on peut taxer ça d'égoïsme et je m'efforce réellement de penser un peu plus aux autres ; mais parfois c'est vraiment très difficile. Qu'il s'agisse de dinosaures (ça, c'était quand j'étais plus petit, je m'empresse de le préciser), des Pokémon, des Playstation ou d'ordinateurs ces derniers ont toujours présenté une fascination récurrente pour moi - je sens une telle vague d'excitation monter en moi que je ne peux même pas la décrire. Je dois absolument discuter du sujet. Être stoppé net me met dans un tel état que je peux facilement rentrer dans une rage folle. En écrivant tout cela, je réalise à quel point tout cela peut paraître fou, mais je ne fais que décrire la réalité (Jackson, 2007, p.41).

L'autiste semble cherche à témoigner de ses connaissances relatives à l'intérêt spécifique, mais en fait l'extrême investissement de ce dernier le conduit initialement à fuir l'échange et à se couper des autres. "As-tu remarqué," demande-t-on à I'un d'eux, "qu'au bout d'un certain temps les personnes deviennent lassées et tentent de parler d'autre chose", il réplique: "Je le sais bien et c'est à cause de cela que je n'ai pas d'amis, mais ils doivent m'écouter ou, alors, ils ne sont rien pour moi"? (Lemay, 2004, p.148). Vers dix ans, Tammet n'avait pas même conscience d'importuner ceux à qui il faisait part des ses centres d'intérêt, son témoignage corrobore cependant les précédents:

Quand je parlais à quelqu'un, c'était souvent d'une seule traite, sans m'arrêter. L'idée de faire une pause ou de parler à tour de rôle ne me venait pas. [...] Je ne comprenais pas que le but de la conversation n'est pas de parler uniquement des choses qui vous intéressent. Je parlais avec force détails jusqu'à être vidé de tout ce que j'avais à dire. Je sentais que j'aurais pu éclater si quelqu'un m'avait interrompu. Il ne m'apparut jamais que le sujet dont je parlais puisse ne pas être intéressant pour mon interlocuteur. Je n'ai jamais non plus remarqué s'il commençait à s'impatienter ou s'il jetait des regards autour de lui. Je continuais à parler jusqu'à ce que l'on me dise quelque chose du genre : Il faut que j'y aille, maintenant (Tammet, 2007, p.85). 
L'autiste semble porté à communiquer à partir de son intérêt spécifique; mais en fait les signifiants qui composent celui-ci sont plutôt initialement utilisés pour faire barrage à I'interlocution. Leur intense investissement libidinal est d'abord mis au service de soliloques, témoignant leur nature de $\mathrm{S} 1$ tout seuls.

Bien que les langues privées entrent en résonance avec le corps, suscitant plus que traduisant des émotions, elles ne sont pas des lalangues verbeuses. Ce sont des constructions originales cherchant l'ordre et la clarté. Elles sont impropres à l'échange, elles ne reposent pas sur une cession de la voix, elles n'incluent pas la fonction du signifiant-maître: tantôt Tammet ignore d'où en vient la signification, tantôt il l'invente, dans les deux cas elle s'avère solipsiste, elle ne vaut que pour lui-même. La langue privée est une langue de S1, elle ne saurait faire lien social, mais ce n'est pas une lalangue inconsistante, en raison d'ébauches de constructions et d'ordonnancements qui lui sont propres.

\section{La langue factuelle de signes figés.}

Malgré l'importance prise par le S1 tout seul dans les propos de l'autiste, ce n'est pas un exilé de l'échange verbal : il n'est ni toujours verbeux, ni toujours hermétique. Williams nous indique deux utilisations possibles des mots, dans la première, celle sur laquelle nous venons de nous attarder, prime une jouissance solitaire du sonore; dans la seconde, ils deviennent, selon son expression, des supports d'accumulation de faits (Williams, 1994, p.169). Ce clivage opéré par les autistes dans le traitement de la parole a souvent été remarqué. La langue verbeuse prédomine chez les autistes de Kanner; tandis que l'autre langue majeure, la langue factuelle, connaît ses développements les plus élaborés chez les autistes d'Asperger. Dans le clivage dont fait état Williams concernant sa construction subjective, la langue factuelle, souvent dite fonctionnelle, est issue de la part d'elle-même qui s'est pliée à l'éducation qui lui fut imposée, c'est-à-dire qu'elle est en prise avec le discours de l'Autre, tandis que la langue verbeuse appartient à son univers personnel "complètement coupé du reste du monde " (Williams, 1992, p.274).

Quelles sont les caractéristiques de "la langue d'accumulations de faits" évoquée par Williams? Partons d'un exemple rapporté par Kantzas. Georges répète une brève historiette:

le chasseur de peaux se lève très tôt le matin. Il sort de sa maison avec son fusil et beaucoup de chiens pour aller dans la forêt. Lorsque les chiens voient le renard ils se mettent à aboyer. Le chasseur épaule alors son fusil, tire et tue le renard. Le chasseur va ensuite au marché pour vendre la peau du renard qui sert à faire des vêtements pour la fourrure (Kantzas, 1987, p.121-122).

Ces successions de faits, sans commentaires, sans affects, semblent viser à une simple 
présentation des choses, sans implication de la voix énonciative. Une autre observatrice de ces phénomènes note que de tels propos s'avèrent essentiellement de "nature constatante" et non intentionnelle. Aubin par exemple, ne pouvait dire que l'un de ses camarades avait été puni par la maîtresse parce qu'il avait été méchant. Les remarques de cet enfant, rapporte B. Donville, "se cantonnaient au détail, mentionnant la couleur du manteau d'un camarade, remarques qu'il sortait d'ailleurs inopinément, alors qu'on ne lui demandait rien de particulier". On note la subsistance d'un élément de soliloque dans des propos néanmoins adressés.

Il se plantait là tout bonnement devant sa mère pour l'en informer, puis se taisait sans ajouter quoi que ce fut d'autre. Rien dans son langage ne racontait, ne décrivait, ne cherchait à déduire, au mieux on obtenait de lui des considérations ponctuelles dont il ne tirait jamais aucun conséquence... (Donville, 2006, p.68).

De tels propos s'avèrent très différents du verbiage: ils s'inscrivent dans un effort pour communiquer, c'est pourquoi ils doivent être produits dans la langue de l'autre. En outre, la jouissance de la voix s'y trouve gommée, tandis qu'elle s'affirme dans le verbiage.

Une des particularités de la langue factuelle des autistes, soulignée par tous les spécialistes, réside dans l'emploi massif de substantifs, catégorie linguistique qui exprime simplement l'existence des choses. L'ancrage dans la concrétude dont elle témoigne semble provenir du souci de n'utiliser essentiellement que des mots ayant pour référent un objet cernable dans la réalité. Une observatrice attentive du langage de sa fille autiste, Clara Park, précise:

qu'ils soient concrets ou abstraits, elle saisissait immédiatement tous les mots absolus, ceux qui exprimaient des concepts définissables et compréhensibles en eux-mêmes: "boîte", "chat", "girafe", "rectangle", "chiffre", "lettre". En revanche, elle était incapable de comprendre les mots de relations, ceux qui ne trouvent leur plein sens qu'au sein d'une situation où l'élément humain joue un rôle [...] (Park, 1972, p.216).

Il existe en effet beaucoup de mots qui nécessitent une appréhension d'un contexte et une mise en relation avec d'autres mots pour pouvoir être compris. On ne peut par exemple cerner ni petitesse ni grandeur absolue. En fait, depuis Saussure, on sait que "dans la langue il n'y a que des différences": le signe, le signifiant et le signifié ne se définissent que dans des systèmes d'oppositions différentielles et interdépendantes. Il en résulte que la signification d'un élément n'advient que de sa mise en relation avec d'autres, laquelle implique un travail subjectif, un exercice du jugement, auxquels l'autiste de Kanner ne se risque pas. Il s'oriente vers un langage qui décrirait les faits sans que lui-même ait à les interpréter. Dès lors, son idéal serait un code qui 
parviendrait à connecter les mots de manière constante et rigide à des objets ou à des situations clairement déterminés.

Ce n'est pas la complexité d'une langue qui pose problème aux autistes, explique $\mathrm{K}$. Nazeer, en fait il est probable qu'elle les aide plutôt, dans la mesure où plus il y en a, moins un mot risque d'être polysémique. Plus il y a de règles et de structures, et moins un autiste doit se reposer sur son intuition et sur le contexte (Nazeer, 2006, p.26).

L'idéal pour eux, souligne-t-il, serait "un sens/un mot", c'est-à-dire une langue qui se réduirait à un code, dès lors totalement construite avec des signes.

\section{Ancrage du signe dans la situation d'apprentissage.}

Une des caractéristiques initiales de la langue factuelle réside dans la permanence de la situation originale d'apprentissage. Kanner note d'emblée le phénomène. Le père de Don, rapporte-t-il, essayait de lui enseigner l'usage du "oui" et du "non". Il lui demanda: "Veux-tu que je te prenne sur les épaules?". Don répéta la question comme en écho, ce qui signifiait son accord. Le père lui dit alors : "Si tu le veux, dis 'oui', si tu ne le veux pas, dis 'non'". Don prononça le "oui" attendu par son père, qui le prit sur les épaules. Mais hélas, Don, pendant des années, ne dit jamais "oui" qu'en tendant les bras vers les épaules de son père. "Oui" ne voulait dire que cela" (Kanner, 1983, p.221).

Les autistes de Kanner, constate Grandin, "sont incapables d'assimiler le moindre écart par rapport aux images qui sont emmagasinées dans leur mémoire" (Grandin, 1997, p.184). Il arrive qu'ils créent des mots qui ne sont compréhensibles que si l'on a connaissance de la situation première qui leur a donné naissance. Lors du développement du langage de son fils, Thomas Hilde De Clercq fut surprise de constater qu'il inventait des mots pour nommer des objets qui avaient la même fonction, mais qui n'avaient pas exactement la même apparence. Il usait par exemple de différents noms pour désigner une "bicyclette". "Il avait une "bicyclette", un "tracteur", des "roues dans la boue", des "roues dans l'herbe" et "des petits pieds sur les pédales". Tout le monde le trouvait très créatif, mais j'avais des doutes, commente sa mère, je pensais qu'il ne pouvait pas faire autrement. Lorsque je lui disais : "Va faire un tour à bicyclette", il ne comprenait pas, car à ce moment-là, il ne voyait que les "petits pieds sur les pédales". Pour lui le mot bicyclette n'avait pas encore de signification généralisée (De Clerq, 2005, pp.22-23).

En raison d'une adhésivité des termes et des expressions de la langue factuelle à la situation première dans laquelle ils ont été acquis, leur signification tend à rester figée. Une telle langue procède à un codage de la réalité. Elle réalise l'idéal : un mot/un sens. Non seulement le mot devrait être univoque, mais les choses elles-mêmes ne devraient pas changer de dénomination. 
Les enfants, constatent les linguistes, apprennent vite qu'une chose peut avoir plusieurs noms, que le chien est un animal, et peut s'appeler Médor et Toutou. Cela ne semble pas leur poser de problèmes. "Ils s'attendent à ce qu'un papa soit aussi un monsieur et qu'il s'appelle aussi Pierre ou Paul ou 'mon chéri'"' (Boysson-Bardies, 1996 p.158). Or, cela ne va pas de soi pour un enfant autiste. Pour eux, que le signe soit sonore ou scriptural, il reste longtemps corrélé à une expérience déterminée. C'est ce qui explique que la formidable mémoire musicale de tel autiste bute toujours sur la même erreur inhérente à la première audition, ou qu'une faute d'orthographe soit toujours reproduite parce que présente dans le premier texte où le mot fut rencontré. La difficulté de l'autiste à généraliser s'ancre dans la rigidité du signe lesté par la persistance de la situation d'apprentissage, de sorte qu'il est peu apte à se modifier quand le contexte change. "Si j'apprenais quelque chose debout avec une femme un jour d'été", relate Williams, "la leçon n'évoquait rien si je me trouvais dans une même situation dans une autre pièce avec un homme un soir d'hiver" (Williams, 1994, p.91). De ce fait, même captée dans la langue de I'Autre, la langue factuelle reste compatible avec de radicales incompréhensions dans l'échange résultant d'une prise au pied de la lettre de l'information.

Un jour qu'on me sermonnait pour avoir fait des graffitis sur les murs du parlement pendant une excursion, rapporte Williams, je promis de ne plus recommencer. Dix minutes plus tard, on me surprit à récidiver, sur le mur de l'école cette fois. Je n'eus pas le sentiment d'avoir désobéi ni failli à ma promesse pour le seul plaisir de faire le clown : ce qui était valable pour les murs du parlement ne l'était pas pour moi pour les murs de l'école. Je n'avais pas fait exactement la même chose qu'auparavant. C'était tout. (Williams, 1992, p.115).

Les autistes butent longtemps sur les signes qui ne peuvent être rapportés ni à un référent concret ni à une image. Chacun constate que les termes syntaxiques et les conjugaisons leur sont d'un abord difficile. Dès que la notion décolle du référent pour passer d'un élément à une généralité, la compréhension de l'autiste rencontre un obstacle:

j'ai toujours eu la plus grande difficulté, confie Williams, à concevoir la transformation d'une chose en une autre. Je savais ce qu'étaient les vaches, mais quand elles devenaient un troupeau, elles cessaient pour moi d'être des vaches. Je comprenais bien que le mot 'troupeau' désignait un ensemble mais je n'avais, par contre, aucune idée de ce que pouvait signifier le mot 'bétail'. Même chose pour la 'fourrure'. Une fois cousue, ça n'était qu'une sorte d'étoffe qui n'avait jamais été un animal et n'aurait pu l'être (Williams, 1994, p.133). 
On conçoit que les termes polysémiques leur soient d'un abord difficile.

\section{La non incorporation du signe de la langue factuelle.}

L'adhésivité du signe au référent le rend impropre à coder les affects, qui s'expriment différemment chez chacun, qui possèdent des nuances, qui sont souvent fugitifs et changeants, et qu'il est difficile d'objectiver. Il est notable que les premiers mots expressifs des enfants autistes soient le plus souvent des termes qui désignent des objets et non des manifestations émotionnelles. De tels enfants, précise Williams, "sont secrètement piégés dans une affectivité mutilée ; ils ont des sentiments et des sensations, mais qui se sont développées dans l'isolement. Ils ne peuvent pas les verbaliser de façon normale "(Williams, 1994, p.301). "Le cerveau", confirme Harrisson, "ne reçoit pas les messages du corps, même si le cerveau et le corps font leur travail chacun de leur côté " (Harrisson, 2010, p.311). Il en résulte un manque de spontanéité et d'initiatives personnelles qui contraignent les autistes à observer les comportements sociaux pour tenter de remédier à la coupure entre leur intellect et leur être de jouissance.

Enfant [relate Grandin], j'étais comme un animal sans instinct pour se guider ; je devais tout apprendre par approximations successives. Je passais mon temps à observer, à chercher le meilleur comportement possible, mais ça ne collait jamais. Il fallait que je pense à chaque interaction sociale (Grandin, 1997, p.154).

Devenue adulte, Grandin n'hésite pas à comparer sa manière de penser à celle d'un ordinateur.

J'ai récemment assisté, rapporte-t-elle en 1995, à une conférence où une sociologue a affirmé que les êtres humains ne parlaient pas comme des ordinateurs. Le soir même, au moment du dîner, j'ai raconté à cette sociologue et à ses amis que mon mode de pensée ressemblait au fonctionnement d'un ordinateur et que je pouvais en expliquer le processus, étape par étape. J'ai été un peu troublée quand elle m'a répondu qu'elle était personnellement incapable de dire comment ses pensées et ses émotions se raccordaient. Quand elle pensait à quelque chose, les données objectives et les émotions formaient un tout. [...] Dans mon esprit, ils sont toujours séparés (Grandin, 1997, p.162).

Une autiste Asperger utilise une formule frappante pour décrire un constant travail d'intellection coupé du vivant : il lui faut en permanence pour s'orienter dans la vie sociale "penser sa vie et vivre sa pensée " (Damaggio, 2011, p.168).

Tous les autistes ayant un recul suffisant sur leur fonctionnement constatent comme Tammet que pendant longtemps ils n'ont pas compris leurs émotions. "C'étaient des choses qui 
m'arrivaient, affirme-t-il, c'est tout, venant de nulle part" (Tammet, 2007, p.118). Ils insistent sur leur incapacité à la saisie et à l'expression de la jouissance et de la vie émotionnelle par le langage. "Je pouvais dire ce que je pensais", affirme Williams, "[...] mais pas ce que je ressentais [...] J'étais émotionnellement constipée" (Williams, 1992, pp.88-89).

Initialement, les émotions éprouvées par l'autiste ne sont pas interprétées par le signe. "Mon visage se mouilla", relate Tammet, suite à la mort de son chien, "et je sus que je pleurais" (Tammet, 2007, p.165). C'est dans un second temps et dans un rapport d'extériorité au corps que l'émotion prend sens. Très exactement ce que décrit Harrisson: "Les autistes ont des émotions mais ils doivent importer le sens de leurs émotions à partir de l'extérieur pour y avoir accès consciemment " (Harrisson., 2010, p.241). Il en résulte que les émotions sont apprises de manière intellectuelle.

Je veux que vous me montriez des émotions, [demande D. Williams à une famille d'amis]. [...] Grâce à des lignes et à des schémas, je vis l'échelle courroucée, l'échelle heureuse et l'échelle triste. Sur ces lignes, ils marquèrent les variantes inférieures et supérieures: fatigué, occupé, énervé, agité, agacé, courroucé et furieux. Ils essayèrent de me montrer comment chaque état pouvait se traduire sur un visage ou se refléter dans des actions" (William, 1994, pp.161-162).

La comparaison avec l'assimilation d'un logiciel s'impose fortement à eux pour souligner l'acquisition d'un savoir ready-made coupé de leur ressenti. "Comme des fichiers informatiques", note Williams, "on peut mentalement mémoriser des jeux d'émotions, les extraire et les interpréter". Cependant, elle constate les limites de ce processus imitatif quant à son appréhension subjective.

Le jeu, souligne-t-elle, n'en est pas pour autant lié à un sentiment réel et on ne comprend pas nécessairement l'émotion représentée, hormis le mécanisme du mode et parfois du moment de son imitation. [...] Une idée n'est jamais un sentiment, simplement le souvenir ou le répertoire mental mémorisé de son impression (William, 1994, pp.288-289).

Dès lors, la non incorporation de la langue factuelle, comme le hors-sens de la langue verbeuse, viennent à l'appui de la thèse des Lefort selon laquelle "dans la structure autistique, le signifiant manque à devenir corps et manque ainsi à faire affect" (Lefort \& Lefort, 2003, p.87).

\section{La difficile contextualisation du signe}

Les signifiants sont pris d'emblée dans une chaîne qui les organise ; tandis que les signes de la langue factuelle sont initialement appréhendés au un par un. Quand la perception est 
structurée par cette langue, elle manque de cohérence, elle apparaît comme "un puzzle". C'est ainsi que je voyais les choses", relate Williams, "bribe par bribe, comme une chaîne d'éléments disparates assemblés arbitrairement les uns aux autres" (Williams, 1992, p.31). Un difficile travail "lui est nécessaire pour qu'elle parvienne à emboîter les morceaux du puzzle" (Williams, 1994, p.121).

Même quand les autistes sont parvenus à une certaine organisation des signes mémorisés, ils décrivent encore souvent leur perception comme "fragmentée". Or, l'autiste d'Asperger cesse d'avoir une attitude passive à l'égard des signes mémorisés : il devient en mesure de les mobiliser pour construire des cohérences locales. Gerland relate ses efforts pour remédier à la fragmentation de son monde en tentant de mettre de l'ordre dans son savoir.

J'avais l'habitude [écrit-elle], d'écrire des étiquettes pour divers objets. Je voulais que tout soit trié, distinct, séparé [...] pour essayer d'ordonner le monde extérieur selon le même système que mon monde intérieur. [...] Il y avait déjà en moi un cloisonnement achevé, muni d'étiquettes pour les actions, les espaces et les univers. En vérité, comme dans un ordinateur ; il y avait beaucoup de ramifications et de bifurcations, mais peu de liaisons transversales. Il était tout à fait clair que le monde qui m'entourait serait plus accessible si je pouvais aussi y faire un tri de ce genre. Je rédigeais donc des étiquettes parlant de la nature de chaque objet et de la place qu'il devait occuper(Gerland, 2004 p.52).

L'appropriation des signes se fait initialement élément par élément. Selon Harrisson, la mémorisation opère à partir $\mathrm{d}$ "'une donnée isolée", ou selon Dawson, elle prend appui sur "un élément insécable d'information" (Mottron, 2004, p.193). Chez les autistes les plus sévères, cet élément peut être constitué par des mots, mais pour les autistes d'Asperger, il prend parfois la forme d'une de ces cohérences locales sur lesquelles Gerland met des étiquettes. L'enregistrement des éléments est plus complexe que celui de photographies: la perception de ces sujets, constate Mottron, "n'est pas statique, elle peut être multimodale (synesthésies) et autorise des manipulations sur le matériel mémorisé" (Mottron, 2004, p.186). Il apparaît en effet que les signes sont rangés dans des ensembles organisés de diverses manières propres à chacun. La cohérence perçue de l'ordre des choses et de ses régularités est I'une d'elles. De surcroît, Grandin confie accorder une place majeure à l'ordre chronologique ${ }^{5}$ pour classer sa "vidéothèque mentale"; d'autres recourent à l'image d'un puzzle en permanente construction, d'autres encore à celle d'une carte géographique, ou d'une banque de données, etc. Il semble qu'il faille entendre par là que chaque terme prend place dans un ensemble, comparable à un fichier, qui permet de le faire entrer en relations avec d'autres fichiers, et que le tout est suffisamment organisé pour que le sujet puisse retrouver l'information.

Harrisson donne une description très détaillée des spécificités de sa mémoire. Elle la décrit 
comme une fragmentation cohérente, qui présente les caractéristiques suivantes:

l'enregistrement de chaque donnée, écrit-elle, est très précis et se fait avec une structure statique. Chaque image est minutieusement différenciée des autres. Tous les détails de chaque donnée sont retenus tels qu'ils sont perçus. Sans nuance aucune. Et chaque donnée est isolée au départ pour ensuite être classée tout en restant indépendante. L'enregistrement est comparable à la réalisation d'un casse-tête. À la fin, on voit toutes les pièces uniques qui nous permettent ensuite de voir l'image globale. Les pièces sont associées et non en interaction. L'image globale du casse-tête - aussi appelé "carte géographique" - s'agrandit au fur et à mesure, selon la quantité de pièces que l'on réussit à attacher, soit les pièces auxquelles on trouve un sens afin de les relier aux autres. Ces petites images globales constituées de plusieurs fragments d'information, très bien définis les uns par rapport aux autres, se fondent dans une grande carte géographique à chaque enregistrement (Harrisson, 2010, p.91).

Ouellette retient l'image du puzzle pour décrire le lent ordonnancement des signes mémorisés opéré par l'autiste de haut niveau.

Ce travail [affirme-t-il], se compare au rassemblement d'un puzzle de mille pièces dont on n'aurait pas le modèle sur la boîte. Au fil du temps des parties sont assemblées, mais l'image globale n'apparaît pas encore. Puis, on finit par avoir suffisamment d"'îles" du puzzle pour reconnaître enfin l'image dans son ensemble et toutes les parties retrouvent enfin leur place (Ouellette, 2011, p.181).

Il est capable de décrire avec une grande finesse des différences majeures de fonctionnement, en distinguant celui du sujet "typique", dont la pensée est commandée par le signifiant-maître et celui du sujet autiste, qui peine à articuler des signes juxtaposés.

D'une certaine manière, note-t-il, la culture neurotypique est ondulatoire, alors que la culture Asperger est corpusculaire. La pensée neurotypique cherche à unir rapidement, presque simultanément, les informations provenant des différents sens et des émotions afin de construire rapidement un sens au monde en vue d'en tirer un avantage à court terme. [...] La pensée Asperger traite plutôt en lente succession ces informations, d'où le fait que les Asperger s'intéressent d'abord aux détails plutôt qu'aux ensembles. Comme un faisceau laser, leur pensée se concentre en premier lieu sur des points. [...] Un Asperger agit ainsi pour laisser le sens émerger progressivement de la lente addition des détails (Ouellette, 2011, p.184). 
Le caractère discret du signe ne donne accès qu'à une mémorisation fragmentée que certains autistes grâce à un travail mnémonique intense appuyé sur du visuel sont capables de rendre plus ou moins cohérent. Les enfants "typiques" n'ont pas cette difficulté. D’emblée, les éléments dont se structurent leur pensée et leur être sont situés dans des ensembles structurés. Les signifiants selon Saussure sont toujours pris dans "une chaîne" (Saussure, 1972, p.103), ils s'assemblent dans une synchronie régie par les lois de la grammaire, de sorte que leur apprentissage emporte spontanément avec lui une assimilation de cette dernière. C'est pourquoi, les linguistes considèrent qu'entre dix-huit et vingt quatre mois les enfants "typiques" sont de véritables "génies grammaticaux". À cet âge, ils fixent le système grammatical qui gouverne leur langue. Or, il est remarquable que le génie grammatical vienne au sujet par exposition au langage et non par apprentissage de celui-ci. Lacan soulignait que ce phénomène fait objection à la psychologie génétique dans son approche du sujet: "l'enfant", constatait-il, "dès ses premières manifestations de langage, se sert de la syntaxe et des particules selon les nuances que les postulats de la 'genèse' mentale ne devraient lui permettre d'atteindre qu'au sommet d'une carrière de métaphysicien" (Lacan, 1966, p.142). Les linguistes s'accordent en effet à considérer que "l'essentiel de la grammaire est connu avant d'être enseigné puisqu'elle est une part essentielle de notre capacité à nous exprimer" (Boyssin-Bardies, 1996, p.218). La plupart des études montrent qu'il y a peu de corrélations entre le langage de la mère et le développement linguistique de l'enfant. Les parents "n'enseignent pas" la langue à leur enfants; ils leur en fournissent des modèles (Boyssin-Bardies, 1996, p.110). Elle ne s'apprend pas comme les tables de multiplication, et son acquisition ne nécessite pas le recours au pédagogue.

Une telle grammaire acquise sans apprentissage, inhérente à l'organisation synchronique de la chaîne signifiante, fait manifestement défaut à l'enfant autiste. Le discours constatant de celui-ci utilise très peu des termes comme "parce que", "car", "par conséquent".

Enfant [relate Grandin], j'omettais des mots comme 'est ', 'le' ou 'ce' parce que, isolés, ils ne signifiaient rien pour moi. De la même façon, des mots comme 'de' et 'un' étaient incompréhensibles. J'ai fini par apprendre à les employer correctement parce que mes parents parlaient bien et que j'imitais leurs tournures de phrases. Encore aujourd'hui, certaines conjugaisons, comme celle du verbe 'être', n'ont aucun sens pour moi (Grandin, 1997, p.33).

L'acquisition des concepts relatifs, note Peeters, est difficile pour les autistes, "car des mots comme 'grand', 'petit', 'large', 'étroit', 'sur', 'de l'autre côté de', 'après', 'donner', 'prendre' prennent leur sens d'après le contexte et par rapport à la relation qu'ils ont avec les autres mots de la phrase". Il note très justement, pour faire comprendre à un enfant autiste les concepts de "grand" et "petit", "il faudrait pouvoir lui communiquer leur signification en partant d'une 
perception "littérale": ceci est "petit", pris au sens absolu, et ici vous voyez le sens invariable de "grand". Malheureusement, cela est impossible" (Peeters, 1996, p.68).

Ce qu'il nomme "perception littérale", nous l'avons souligné, est surtout une perception visuelle, voire tactile. Tous les autistes constatent que les mots qui ne peuvent être rapportés à une image leur sont difficiles à saisir.

\section{La langue factuelle de signes ordonnés.}

Les premières acquisitions du signe s'opèrent de manière statique; cependant, quand les capacités du sujet se développent, il devient peu à peu capable de les mobiliser. "L'autiste", indique à cet égard Harrisson, "n'a pas accès à la mise en ordre par lui-même, sauf s'il a atteint un niveau assez élevé (une fois qu'il a accès au mouvement, à l'interaction) " (Harrison, 2010, p.85) . Il s'avère alors en mesure de les décoller de leur connexion rigide au référent. En interagissant les signes ordonnancés deviennent moins figés et tendent à se détacher de la situation d'apprentissage. Ce ne sont plus seulement des termes-étiquettes, le sujet les organise dans sa mémoire d'une manière qui lui est propre, et qui lui permet de les faire entrer dans des relations d'oppositions mutuelles. Quand Grandin est obligé de recourir à des icônes telles qu' "une colombe ou un calumet" pour se représenter la notion abstraite de paix, ce n'est pas seulement par couplage de ces mots à l'image d'un référent : elle les insère dans des relations d'oppositions avec d'autres oiseaux pour la colombe, et pour le calumet avec d'autres objets qui se fument.

La fixité initiale de la signification du signe tend à s'atténuer progressivement à l'aide d'un intense travail de mémorisation. Grâce à celui-ci advient une certaine souplesse de son usage. Non sans résistance, l'autiste acquiert la notion qu'un même objet peut être désigné par des signes différents, que la localisation de l'objet n'est pas rigide, voire qu'il convient de distinguer la chose et son image.

Mohamed se fâcha [rapporte à cet égard F. Hody], parce que nous persistions à dire 'voiture' plutôt qu' 'auto'. Il lui fallut une nuit pour admettre que deux mots différents pouvaient désigner le même objet. Il nous explique alors qu'on peut dire 'auto' et 'voiture' et 'c'est la même chose'. Un claquage de porte, ajoute-t-on, et cinq minutes de bouderie suffiront à accepter qu'il y ait des girafes au zoo et en Afrique, mais ce sera ensuite tout aussi difficile pour les zèbres. Pour les lions sur les Peugeot, c'est Wanda qui lui prêtera la solution : ce n'est pas un lion mais le 'dessin' d'un lion (Hody, 2008, p.174).

L'ordonnancement mnémonique des signes est organisé chez l'autiste selon une logique spatiale: les modèles évoqués sont la carte géographique, le puzzle, un rangement de CD-rom et un cloisonnement muni d'étiquettes. La structuration de la mémoire résulte d'un effort volontaire, elle s'organise à partir du visuel et elle n'est pas commandée par des éléments inconscients. Le 
sujet affirme être en présence immédiate du rangement des informations mémorisées. Les éléments de sa mémoire restent ancrés dans la perception. En revanche, la mémorisation qui s'appuie sur le signifiant n'est pas visualisable: elle est régie par une grammaire préconsciente, ainsi que par la jouissance attachée dans l'inconscient aux signifiants-maîtres. ${ }^{6}$

Faute d'acquisition spontanée de la grammaire inhérente au système signifiant, les autistes sont contraints de l'apprendre pour l'essentiel par cœur. Ils y parviennent parfois fort bien. Leurs capacités mnémoniques sont telles que beaucoup d'autistes d'Asperger possèdent une excellente maîtrise de leur langue, voire de plusieurs. Ce ne sont pas des génies grammaticaux, c'est précisément pourquoi, afin de compenser la dispersion des signes, ils tendent à devenir des génies mnémoniques.

Il est possible que la propension de l'autiste à la mise en images soit un des éléments qui le porte à représenter le lieu de sa dynamique en un objet qui appareille sa jouissance. Ce dernier est si spécifique que la clinique a imposé l'introduction de nouveaux concepts pour en rendre compte : "I'objet autistique" de Tustin, "l'intérêt spécifique" des modernes Manuels de psychiatrie, et "le bord" pour l'approche lacanienne. Ce dernier constitue la construction défensive majeure pour l'autiste qui cherche à sortir de sa solitude. Avec la rétention des objets pulsionnels et I'aliénation qui s'opère sans la cheville du signifiant-Maître, il caractérise la structure autistique.

\section{Appareillage de la jouissance par le bord}

Les psychanalystes qui ont travaillé avec des enfants présentant des formes sévères d'autisme constatent de manière concordante qu'ils sont souvent préoccupés par les trous, que ce soient ceux de leur corps, ou ceux de leur environnement.

La première enfant autiste qu'il m'ait été donné de rencontrer en hôpital, après avoir fugué dans les couloirs, commençait invariablement les séances en bouchant avec de la pâte à modeler tous les trous de la pièce puis son nombril. Une telle observation n'est pas rare. Les autistes prékannériens sont particulièrement préoccupés par les trous, ceux de leur corps, mais tout autant par ceux de leur environnement. Les trous des W-C ou ceux des lavabos suscitent fréquemment leurs inquiétudes. Éric Laurent souligne une intolérance au trou chez les autistes (Laurent, 2012. p.68). La rencontre de ceux-ci mobilise des angoisses de séparation, d'où leur propension à les boucher; tandis que la mise en jeu d'une perte est ressentie, selon Tustin, comme "un trou noir plein de créatures menaçantes" (Tustin, 1992, p.238). Malika le confirme quand, confrontée à un trou dans une chaise, elle se demande, inquiète, "elle va pas sortir la poupée démontée si tu ${ }^{7}$ ne bouches pas ce trou-là?", de sorte qu'elle s'emploie à le combler avec de la pâte à modeler, en commentant "je bouche le trou pour qu'il n'arrive pas de poupée démontée dedans", elle avait confié en une séance précédente: "perdre son caca, c'est être démontée" et en une autre s'interrogeait: "Quand elle est démontée la poupée, elle a des trous ?" Malika n'avait pas élu d'objet autistique. Pourtant, la pâte à modeler utilisée comme bouche-trou semble s'apparenter à la fonction carapace de cet 
objet tel que le concevait Tustin.

\section{Naissance du bord}

L'insistance de cette psychanalyste anglaise sur un processus d'encapsulement à l'aide de constructions d'objets produits par l'enfant autiste a incité Éric Laurent dès 1992 à considérer que le retour de la jouissance sur un bord (Laurent, 2012. p.68) constituait un élément majeur du fonctionnement autistique. Cependant l'appréhension de l'objet autistique par Tustin comme faisant obstacle à toute assomption de la perte l'a conduit logiquement à considérer celui-ci comme un objet pathologique. Or, beaucoup d'incarnations du bord, voiture, ventilateur, machine à laver, clefs, etc., n'induisent pas à privilégier l'image de la carapace. En fait, il semble même que celle-ci risque d'induire en erreur.

Saisir la naissance clinique d'un bord est un moyen privilégié pour discerner ses fonctions. À cet égard, Bettelheim et Tustin ont recueilli deux documents exceptionnels. Le premier note comme décisif dans la progression de Laurie, une poupée flasque et mutique de sept ans, le moment où elle commença à déchirer du papier en longs rubans et à en faire des frontières. $\mathrm{Ce}$ "furent ses premières activités spontanées, délibérées et, surtout, symboliques. Elles étaient vraiment son invention, sa création à partir de matériaux externes, afin de maîtriser les tensions internes" (Bettelheim, 1969, p.188). Cette activité débuta un jour immédiatement après qu'elle fut allée à la selle. Durant des heures, à partir de feuilles de papier, elle produisait de longs rubans en déchirant concentriquement à partir d'un bord jusqu'à ce qu'elle atteigne le centre. Elle perfectionna ce procédé en commençant par un découpage du centre suivi de son rejet accompagné d'une expression de dégoût. ${ }^{8}$ À l'aide de ces rubans de sa fabrication, elle créait des frontières entre son monde à elle et le reste du monde. Le bord ainsi créé se fonde sur une perte maîtrisée, qui permet de générer un objet protecteur utilisé, non comme carapace, mais comme frontière. Il ne bouche pas un trou: il le cerne.

Le procédé utilisé par David, I'enfant carapace de Tustin, est assez similaire. Il s'agit d'un enfant plus âgé que Laurie, il a quatorze ans, de sorte que la mise en image s'avère plus élaborée. La création s'étend sur de nombreuses séances, mais elle répond à la même séquence que précédemment. Le phénomène prend sa source dans la cession d'un objet corporel, les selles pour Laurie, le pus d'un abcès pour David. Afin de traiter ce trou angoissant, la première opère la perte d'un objet "dégoûtant", le second concrétise l'objet qui en sort, en confectionnant "un monstre aux yeux de mort". Dans les deux cas, le phénomène de corps initial, la cession angoissante, se déplace en une situation qui permet de maîtriser un trou inquiétant par la production d'un objet protecteur. Au terme, I'une érige une frontière protectrice directement issue du trou ; tandis que l'autre confectionne une armure pour se protéger "du monstre au trou" (Tustin, 1972, p.72). Malgré ses préventions à l'égard de l'objet autistique, Tustin note que le fait de la revêtir constitua un "progrès" dans la cure (Tustin, 1977, p.168). Or, quand on examine cette armure en carton, 
dont les photos sont insérées, il apparaît que ce n'est pas une carapace compacte : elle est constituée de deux parties, un visage et une main, elles sont creuses, non reliées entre elles, tandis que le visage porte une trace de trous, puisqu'il comporte des yeux, une bouche et des oreilles. Tustin ne s'attarde pas sur le fait que cette armure est poreuse. Une des fonctions initiales du bord est certes de protéger du trou, mais non pas en le bouchant, le but est plutôt de le circonscrire. En cernant le trou réel, le bord permet de transmuer celui-ci en un manque, moins inquiétant, avec lequel le sujet peut composer.

En localisant sa jouissance sur un objet externe, le sujet autiste témoigne, comme tout être parlant, qu'il entretient une relation dérangée avec la jouissance de son propre corps. Cependant, faute de disposer de la fonction du signifiant unaire, il ne la chiffre pas en un index de l'objet perdu : il s'en sépare par la production d'un objet concret. Les phénomènes de corps qui précèdent la construction du bord de Laurie et de David, à savoir la défécation pour l'une, l'extraction du pus pour l'autre, sont déplacés et maîtrisés par la construction et la manipulation d'un objet externe. En ces circonstances, l'événement traumatique n'est pas chiffré par un événement de corps; ce qui conduit à souligner que le bord n'est pas un sinthome. Il repose sur une première soustraction de jouissance qui introduit dans l'économie du sujet ce qu' Éric Laurent a nommé un en-forme, non pas de l'objet $a$, mais d'un objet de jouissance qui reste présent. Le bord opère une coupure dans la jouissance, il met celle-ci à distance et il instaure un lien du sujet à un objet. C'est un opérateur de capture et de traitement de la jouissance, incarné dans un objet concret, dans une image, dans une personne, ou dans un ensemble thématique de signes. Si le psychotique a l'objet dans sa poche, l'autiste, lui, conserve l'objet séparé à sa main, logé dans un en-forme.

Beaucoup d'intérêts spécifiques semblent répondre à la même logique que celle des premiers bords : ils tirent leur source d'un trauma à l'égard duquel ils développent un savoir protecteur. Un enfant autiste ayant assisté à la mort de son grand-père par arrêt cardiaque pendant une promenade, ne sembla pas pleurer cette mort, mais développa un intérêt pour les maladies cardiaques et lu tous les livres qu'il put trouver sur les pathologies du cœur.

Trois éléments souvent intriqués sont constitutifs du bord : l'objet autistique, le double et l'intérêt spécifique. La machine de Joey, censée lui fournir l'électricité qui l'anime, est à la fois un objet autistique, dont il ne peut se séparer, un double, lui-même étant une machine et la source de son intérêt spécifique, puisqu'il deviendra électricien. Un point commun majeur réside dans l'exceptionnel investissement libidinal dévolu à chacun des éléments. Tous sont l'objet d'une intense passion.

La critique majeure des cognitivistes à l'égard de l'approche psychanalytique consiste à souligner que l'enfant autiste s'intéresse beaucoup à son environnement, même s'il le fait de manière latérale, de sorte que l'autisme ne saurait être appréhendé comme un processus de retrait social. La rétention des objets pulsionnels objecte à cette critique, il est peu contestable qu'il existe 
bien une fuite initiale des interactions sociales ; en revanche, il est exact que la création du bord n'est pas au service d'un processus d'encapsulement. Les témoignages des enfants autistes convergent pour affirmer qu'ils souffrent de leur solitude et qu'ils sont au travail pour tenter de rejoindre le monde.

\section{Le bord centre moteur de la jouissance.}

Le retour de la jouissance sur le bord, selon l'expression d'Éric Laurent, consiste en une sorte de détournement sur cet objet d'une jouissance en excès qui produit alors une animation du sujet. Pendant plusieurs années, il a fallu que Joey soit branché sur sa machine-auto, à l'École Orthogénique de Chicago, pour pouvoir fonctionner, grâce à l'électricité qu'elle était supposée lui fournir. Avec l'aide de celle-ci, il parvenait à appareiller la jouissance pulsionnelle: elle rendait possible la défécation en chauffant les selles, elle régulait l'alimentation quand elle l'accompagnait au réfectoire, elle était porteuse d'un haut-parleur pour traiter la voix et elle comportait de nombreuses ampoules électriques qui captaient le regard. En se donnant l'illusion d'être branché sur une machine, ou sur un double, l'enfant autiste conforte son sentiment de n'être pas vivant, de n'avoir pas d'énergie propre et se décharge d'avoir à prendre des décisions. Les émotions ressenties par l'autiste, n'étant pas interprétés par le signe, sont appréhendées comme la montée d'une houle angoissante. Se faire la marionnette d'un bord qui capitalise la jouissance est une manière de tenter de s'en délivrer. Plusieurs ont expliqué avoir voulu être une machine ou un robot pour ne pas éprouver d'émotions.

Du fait de la non-cession des objets pulsionnels, la dynamique désirante ne se construit pas, ce qui incite parfois des observateurs à faire état d'une "maladie de la volonté" (Park, 1972, p.283). L'autisme, affirme Donna Williams, avait précédé pour elle le moindre souhait personnel. "Mes premiers 'souhaits', rapporte-t-elle, furent copiés sur ceux d'autrui (souvent inspiré par la télévision). Enfant, elle s'éprouvait comme sans fondation, de sorte qu'elle était semblable à un sujet sous hypnose, totalement ouverte à une programmation, ou à une reprogrammation, sans question ni identification personnelle (Williams, 1994, p.124).

Dans les cures individuelles d'autistes sévères, quand le thérapeute parvient à se faire accepter, l'enfant le place en centre moteur de sa jouissance, il instaure une relation fusionnelle avec lui, de sorte qu'il devient un double dynamique.

Peter, un enfant autiste d'une dizaine d'années, d'après sa thérapeute "vivait toute relation dans l'abandon de son identité et la fusion avec l'autre personne " (Rothenberg, 1979, p.246).

Au début [écrit Mira Rothenberg], j'étais sa force, sa santé, son contact avec la réalité, son créateur et son sauveur. [...] Il était vis-à-vis de moi dans un état de profonde et totale dépendance [...] Je lui donnais mon énergie et le laissais dépendre de moi et se nourrir de ma force [...] Jamais il ne voulait assumer aucune responsabilité dans la vie, comme s'il ne 
s'intéressait qu'à son monde imaginaire. Quand je lui demandais de choisir, il répondait toujours : 'Qu'est-ce que Mira préfère?' [...] Même dans la souffrance, il refusait toute responsabilité. Il criait, pleurait et il fallait être un fin détective pour arriver à déceler où il avait mal. Jamais il ne le disait. Il répétait souvent : Mira doit décider pour Peter. Cela lui fait du bien. Cela le met plus à l'aise (Rothenberg, 1979, p.277-279).

Cette vignette clinique met particulièrement en évidence que le traitement du manque est remis au bord, protégeant ainsi le sujet de prendre le risque d'engager son désir.

Le branchement devient plus discret et moins permanent à mesure que l'on progresse sur le spectre de l'autisme vers le pôle où celui-ci devient parfois socialement invisible. Néanmoins, une autiste suédoise de haut niveau, telle que Gunilla Gerland, relate avoir encore besoin d'un appui sur un bord pour initier ses conduites.

Très souvent, écrit-elle, c'est plus facile si j'ai quelqu'un avec moi lorsque je dois faire quelque chose pour la première fois, car je peux, en quelque sorte, me fier à son système nerveux. À cet effet, je me suis presque servi des gens. J'ai pu faire semblant de demander leur compagnie alors qu'en réalité, j'avais besoin d'une escorte. Je me suis arrangée pour emmener quelqu'un avec moi pour visiter un musée ou une galerie où je n'étais jamais allée auparavant dans le seul but de pouvoir y retourner seule plus tard sans difficulté. Parfois, j'aurais aimé, telle qu'un ordinateur dans un réseau, pouvoir me brancher sur le système nerveux de quelqu'un d'autre pour m'en servir dans de telles circonstances, au lieu de devoir l'emmener avec moi, et d'être obligée de le rencontrer et d'agir en même temps (Gerland, 2004. P.232).

Le recours que Temple Grandin devait faire à sa trappe à serrer était lui aussi intermittent. Il suffisait qu'elle se loge en celle-ci pendant un certain temps pour y obtenir une régulation de sa jouissance. La construction de ce bord complexe mérite que l'on s'y attarde parce qu'il semble bien mettre en image l'encapsulement autistique conçu par Tustin. En première analyse, le séjour en cette trappe, dont les parois devaient enserrer étroitement Grandin, semblait permettre d'opérer un retour à la jouissance fusionnelle partagée qui caractérise le lien aux bords initiaux. Or, elle confie que cette machine prend sa source dans "une sorte de boîte ressemblant à un cercueil" (Grandin, 1994, p.51), et elle tire son modèle de ce dont elle est devenue une spécialiste mondiale, à savoir selon ses propres propos, "une des machines à tuer les plus performantes au monde"(Grandin, 1997, p.238). Grandin ne fait pas mystère qu'elle se loge en celle-ci comme le fait une vache et que cet animal est pour elle un double. Or, quand il en sort, c'est pour aller à la mort. Que cette trappe ait pour fonction de traiter le trauma de la mort par les sorties réitérées de sa protection, Grandin n'est pas très loin de le concevoir. 
La plupart des gens, écrit-elle, ne se rendent pas compte que la mort dans les abattoirs est beaucoup plus douce que la mort naturelle. Les animaux sauvages meurent de faim ou bien sont victimes des prédateurs ou bien dépérissent à cause du climat. Si j'avais le choix, j'aimerais mieux passer par un système d'abattage industriel plutôt que de me faire arracher vivante les tripes par les coyotes ou les lions (Grandin, 1997, p.238).

Une des caractéristiques de l'autiste de haut niveau est d'être parvenu à un évidement de la jouissance excessive du bord. Les coupures réitérées opérées par Grandin dans la fusion restaurée avec le bord semblent y avoir contribué. Ce n'est que tardivement, en 2010, qu'elle mentionne que sa machine s'est cassée et qu'elle ne l'a pas réparée. Subsistent malgré tout deux intérêts spécifiques, qui médiatisent ses rapports sociaux, l'un pour les trappes à bétail, l'autre pour le savoir sur l'autisme.

\section{L'évidement du bord.}

L'évidement du bord se produit à l'occasion de décisions assumées par l'autiste de procéder à une perte portant sur celui-ci. Sa propension à recourir aux images lui permet parfois de la mettre en scène. La cession de jouissance peut encore advenir quand le sujet prend le risque de se défaire temporairement de son système de protection à l'égard des échanges sociaux; ce qui équivaut à un désinvestissement partiel de la fonction du bord.

Williams souffrit longtemps de la présence de ses compagnons imaginaires qui lui permettaient certes une adaptation sociale mais qui faisaient persister un certain vécu de "mutilation psychique" quand elle se branchait sur eux. C'est pourquoi, elle discerna que son évolution devait passer par la disparition de la "partie d'elle-même" qu'ils représentaient. À l'adolescence, elle tenta de se séparer de Willie. Je décidai de tuer Willie, écrit-elle, cet autre moimême toujours en colère.

On m'avait donné [relate-t-elle], une poupée figurant un petit garçon vêtu d'un jean et d'une chemise. Je l'enroulai dans un morceau de tissu rouge écossais, une étoffe qu'affectionnait ma grand-mère. [...] Je me procurai une petite boîte en carton que je peignis en noir. J'attendis qu'il n'y eût plus personne à la maison, puis je partis vers l'étang aux poissons où j'immergeai ma personnification symbolique de Willie dans son noir cercueil, effaçant minutieusement toute trace des funérailles (Willians, 1992, p.113).

Carol et Willie, ces deux éléments du bord de Williams, lui fournissaient une "protection anesthésiante" (Willians, 1994, p.159) qui lui permettait de "supprimer"l'émotion (Willians, 1992, p.143). Le meurtre imaginaire de Willie ne fut pas immédiatement réalisé: il fallut encore de 
longues années avant que sa disparition ne soit assumée, mais il révèle comment un autiste de haut niveau parvient à traiter son bord : par un vidage progressif de ce qui le constitue par lequel s'atténue la jouissance qui y est attachée.

Williams souligne l'importance que prit pour elle la cession de l'un de ses bords, son premier livre, en acceptant de s'en séparer par la publication. La nouvelle de l'acceptation de son manuscrit la plongea dans une vive inquiétude.

Les vies de Carol, de Willie et la mienne allaient être exposées à travers le monde. J'étais la plus évasive des personnes que je connaisse et j'allais être sous peu la plus publique. [...] J'avais peine à croire qu'il s'agissait de 'mon' livre. Je perdais tout contrôle; d'autres allaient lire mes mots. J'eus envie de retrouver chaque exemplaire, de les déchirer et d'en brûler les morceaux (Willians, 1992, p.124).

Décisive aussi fut pour elle la découverte tardive que pour penser et ressentir, "une chose devait avoir un système nerveux" (Willians, 1992, p.100). II lui fut très difficile d'accepter que les objets soient morts, "sans connaissances, sans sentiments, sans volition". Ce vidage de la jouissance du bord, qu'elle associe à la disparition de ses compagnons imaginaires, lui donna le sentiment d'être délaissée par les choses qui lui avaient apporté la sécurité. Elle persista cependant à tenir quelques objets à l'écart de cette nouvelle logique matérielle, en particulier deux peluches baptisées "Ours Orsi" et "Chien voyageur". Elles lui servaient à établir "des ponts avec le monde extérieur" (Willians, 1992, p.100). Indice que l'évidement du bord est un travail progressif qui s'opère par une réitération de cessions de jouissance.

Ce processus peut s'insérer dans la cure d'un enfant autiste et y introduire des scansions décisives. Dibs en produit une représentation quand il achève sa psychothérapie par une capture de sa voix et une mise en scène de sa perte. "Écoute-moi bien, magnétophone", dit-il. "Tu vas attraper et garder ma voix. [...] Je vais faire un long enregistrement et nous le garderons pour toujours et pour toujours. Ce sera juste pour nous deux" " (Axline, 1967, p.197). Au terme, il insiste sur le fait que sa psychothérapeute doit garder l'enregistrement: "rangez cette bande, lui dit-il, mettez-la dans la boîte et rangez-la, et gardez-la juste pour nous deux" (Axline, 1967, p.199). Cette assomption d'une cession de jouissance s'accompagne d'une mise en jeu de la dimension du manque dans son rapport à l'Autre. Il sait que les vacances d'été vont interrompre sa psychothérapie qui touche à sa fin. Vous allez me manquer, dit-il à sa thérapeute. "Ça va me manquer de ne plus venir. Il ajoute : Est-ce que je vais vous manquer?" (Axline, 1967, p.210). Quelque chose peut maintenant manquer au sujet comme à l'Autre, et pourtant ce n'est pas perdu, mais capté par un contenant qui reste accessible. Deux ans et demi après l'achèvement de la cure, quand sa thérapeute rencontre Dibs, elle constate qu'il développe un intérêt spécifique dans le domaine de la botanique, dérivé de l'arbre qui fut l'un de ses objets autistiques. 
L'évolution du travail de Joey, l'enfant-machine de l'École Orthogénique de Chicago, montre une succession d'élaborations du bord, qui passe de machines redoutables à des formes de plus en plus humaines et bienveillantes. Kenrad, qui tend à remplacer la machine pour le traitement de la jouissance anale est encore tout-puissant et dangereux, sa défécation est un événement cosmique inquiétant. Mitchell auquel Joey s'attache ensuite est un garçon protecteur avec lui, dont la défécation ne produit plus explosions et lumières. Valvus le compagnon imaginaire qui leur succède pouvait se régler lui-même, il pouvait s'ouvrir ou se fermer comme une valve quand cela était nécessaire. Avec lui, Joey parvint à un contrôle de sa propre élimination qui l'angoissait tant. Il apparaît nettement que la progression des incarnations du bord se fait par vidages réitérés de la jouissance excessive attribuée à celui-ci. Au terme, il semble qu'en effaçant les doubles, Joey ait pu parvenir à réduire son bord à l'intérêt spécifique que constituaient pour lui les machines électriques, puisqu'il avait fait profession de s'occuper de celles-ci.

Le privilège du signe dans la pensée des autistes induit le développement de capacités mnémoniques exceptionnelles. De leurs talents en ce domaine, certains font un intérêt spécifique dont ils essaient de tirer quelques profits et quelques échanges sociaux. Parmi ses divers métiers "provisoires", Vienamin Cherechevsky tenta de faire une carrière de mnémoniste, en se produisant en spectacle. Or, il lui arriva de se trouver encombré par sa mémorisation sans limite. Ne parvenant pas à effacer les données qui lui avaient été soumises lors de séances rapprochées, il craignit qu'elles ne se superposent et le conduisent à faire des erreurs. Il tenta alors de les mettre par écrit et de brûler les bouts de papier sur lesquels il inscrivait ce qu'il devait oublier. Or, cette perte concrète mise en images ne fonctionna pas.

J'avais donné ce soir-là, rapporte-t-il, trois séances consécutives. Je me sentais fatigué physiquement et me demandais comment mener une quatrième séance. Je m'attendais à voir surgir les chiffres des trois séances précédentes... J'étais dans l'angoisse... Verrai-je dans un instant apparaître le premier tableau? Je l'appréhendais. Je me disais : je veux je ne veux pas... Et pendant ce temps le tableau n'apparaissait toujours pas. La raison en est compréhensible : c'est parce que je ne veux pas ! Ah ! ainsi si je le veux il n'apparaîtra pas... Donc il fallait simplement en prendre conscience! (Luria, 1970, p.61).

Chose surprenante, commente Luria, ce procédé s'avéra efficace. Si l'on conçoit qu'il s'agit d'une décision assumée de se détacher d'une partie de son bord, introduisant un manque apaisant en celui-ci, le phénomène apparaît moins surprenant. La modification de la position subjective de Vieniamin qui accompagne ce décollement salvateur semble le confirmer:

Je me suis senti délivré d'un seul coup, constata-t-il. La certitude d'être à l'abri des erreurs me donna de l'assurance. Je parle avec plus d'aisance, je puis me permettre le luxe de 
faire des pauses, je sais que je peux à mon gré empêcher l'apparition des images. Je me sens parfaitement bien (Luria, 1970, p.61).

Il était parvenu à prendre à son compte une part de la jouissance qu'il attribuait à son bord. L'évidement du bord permet aux autistes de haut niveau de remédier au trouble initial de la communication, qui prend sa source dans la rétention des objets pulsionnels. Grandin l'exprime très clairement:

[...] quelque chose, écrit-elle, s'est passé au cours du processus qui a déconnecté le 'fil' dans le cerveau qui rattache un enfant à sa mère et aux autres êtres humains qui lui offrent leur affection. Ce n'est qu'au moment où j'ai été assez grande et assez compétente pour construire la machine à serrer que la connexion a été réparée (Grandin, 1986, p.128).

Si l'accrochage à I'Autre est restauré, il semble cependant qu'il doive persister à être médiatisé par un bord, le plus souvent réduit à l'intérêt spécifique. "L'invention est le seul 'remède' du sujet autiste, souligne Éric Laurent, et elle doit, chaque fois, inclure le 'reste', soit ce qui demeure à la limite de sa relation à l'Autre: ses objets autistiques, ses stéréotypies, ses doubles" (Laurent, 2012, p.65).

Il est souvent difficile d'engager une cure analytique avec un enfant autiste. Le transfert est initialement entravé. Quand un analyste arrive à se faire accepter, par une attitude non intrusive, associée à des conduites en miroir, ou en parallèle, alors un transfert fusionnel s'instaure, l'analyste devient un double. L'évidement du bord conduit par la suite à un décollement du double et à un investissement tempéré de l'intérêt spécifique.

Les angoisses liées à la cession des objets pulsionnels incitent les autistes à une appropriation solitaire du langage, qui conduit à survaloriser dans ce processus les perceptions visuelles et tactiles, ce qui suscite un privilège pour le signe, et ce qui génère un bord qui est une mise en-forme de l'objet de jouissance. Les méthodes d'apprentissage des signes prônées pour la prise en charge des autistes rencontrent une limite quand elles omettent qu'un appui sur le bord est une condition nécessaire pour leur construction subjective. Accepter la mise en place de celui-ci n'est cependant pas suffisant, il convient encore d'accompagner un travail d'évidement de sa jouissance en excès, tout en respectant l'intérêt spécifique.

Que l'évolution du bord se produise par sauts créatifs générés par l'assomption d'une perte, et que les angoisses initiales de l'autiste prennent leur source dans la rétention des objets pulsionnels, sont des notions qui restent nécessairement étrangères aux approches cognitivistes, même aux plus pertinentes qui incitent à s'appuyer sur les "points forts" pour le traitement, c'est pourquoi, n'en déplaise aux spécialistes, l'approche psychanalytique reste incontournable pour s'orienter dans le travail avec les autistes. 


\section{Notas}

1. L'intervention de Jean-Claude Maleval s'est déroulée à Lyon, le mardi 13 mars 2018, dans le cadre de I'ACF-RA. Nous publions ici l'intégralité de la conférence. Elle a été revue et partiellement modifiée par l'auteur.

2. Je remercie V. Gay de m'avoir communiqué un document non publié notant la langue privée de Théo.

3. $1 / \mathrm{Aa} ; 2 / \mathrm{Na} ; 3 / \mathrm{Ya} ; 4 / \mathrm{Ka} ; 5 / \mathrm{Yaa} ; 6 / \mathrm{Si} ; 7 / \mathrm{Sa} ; 8 /$ oui ; 9/Io ; 10/Di ; 11/On ; 12/dou ; 13/ Ttai (soufflé) ; 14/To ; 15/Tin ; 16/Té ; 17/di-sa ; 18/di-oui ; 19/Dio ; 20/va.

4. "Les nombres sont ma langue maternelle, celle dans laquelle je pense et je ressens" (Tammet, 2007, p.15).

5. "Mes souvenirs se présentent toujours dans l'ordre chronologique, et les images sont toujours particulières" (Grandin, 1997, p.29].

6. "L'inconscient a à faire d'abord avec la grammaire, il a aussi un peu à faire, beaucoup à faire, tout à faire, avec la répétition, c'est-à-dire le versant tout contraire à ce à quoi sert un dictionnaire" (Lacan, 04/11/1971).

7. Elle utilisait ici le "tu" en place du "je" comme elle le faisait fréquemment.

8. "Au dernier stade de cette évolution, note Bettelheim, elle déchirait le centre de la feuille de papier dès le début, le jetait, toujours avec une expression de dégoût, et alors seulement déchirait la feuille concentriquement en se dirigeant vers le centre maintenant vide. Il était impressionnant de constater l'adresse avec laquelle elle enlevait, apparemment sans effort, le centre exact de la feuille de papier et avec laquelle elle arrivait toujours exactement en cet endroit lorsqu'elle avait fini de déchirer" (Bettelheim, 1969, p.187).

\section{Referências bibliográficas}

Axline, V. (1967). Dibs. Développement de la personnalité grâce à la thérapie par le jeu (1964).

Paris: Flammarion.

Berquez, G. (1983). L'autisme infantile. Paris: PUF.

Bettelheim, B. (1969). La forteresse vide. Paris: Gallimard.

Blake, J., Boysson-Bardies, B. (1992). Patterns in babbling: a cross-linguistic study, Journal of Child Language, 19(1), 1992, pp.51-74.

Boysson-Bardies, B. (1996). Comment la parole vient aux enfants. Paris: O. Jacob.

Chericoni N., De Brito, Wanderley D., Constanzo V., Diniz-Gonçalves, A., Leitgel, Gille M., Parlato, E., Cohen, D., Apicella, F., Calderoni, S., Muratori, F. (2016) Pre-linguistic Vocal Trajectories at 6-18 months of Age As Early Markers of Autism. Front. Psycholog. $7: 1595$. Doic 
10.3389/fpsyg. 2016. 01595.

Damaggio, N. (2011). Une épée dans la brume. Syndrome d'Asperger et espoir. Paris: Anne Carrière.

Danon-Boileau, L. (2002). Des enfants sans langage. Paris: O. Jacob.

Dechais, A. (2009). Libres propos philosophiques d'une autiste. Paris: Presses De La Renaissance.

De Clerq, H. (2005). Dis maman, c'est un homme ou un animal?. Paris : AFD.

Dovan, J., Zucker, C. (2010, out). "Autism's First Child. Atlantic Magazine". October 2010. http://www.theatlantic.com/magazine/archive/2010/10/autism-8217-s-first-child/8227/

Donville, B. (2006). Vaincre l'autisme. Paris: O. Jacob.

Gay-Corajoud, V. (2018). Nos mondes entremêlés, Imprim'vert.

Gerland, G. (2004). Une personne à part entière. AFD, Mougins.

Grandin, T. (1994). Ma vie d'autiste (1986). Paris: Odile Jacob.

Grandin, T. (1997). Penser en images (1995). Paris: Odile Jacob.

Harrisson, B. (2010). L'autisme au-delà des apparences. Concept ConsulTED, Rivière du loup,

Québec, Canada.

Hebert, F. (2006). Rencontrer l'autiste et le psychotique. Paris: Vuibert.

Higashida, N. (2014). Sais-tu pourquoi je saute? Paris: Les Arènes.

Hody, F. (2008). Pertinence clinique de la distinction de l'autisme. Les Feuillets du Courtil.

Jackson, L. (2007). Excentriques, Phénomènes et Syndrome d'Asperger. Paris: AFD Editions, Mouans Sartoux.

Kanner, L. (2002). "Le langage hors-propos et métaphorique dans l'autisme infantile précoce" (1946), American Journal of Psychiatry, 1946,103, p.242. Tradução de G. Druel et F. Sauvagnat, in Psychologie clinique, 2002, 14, pp. 204-213.

Kanner, L. (1983). "Troubles autistiques du contact affectif". In BERQUEZ G., L'autisme infantile, Paris: PUF.

Kantzas P. (1987). Le passe-temps d'un Dieu. Analyse de l'autisme infantile. Paris: Dialogues.

Lacan, J. (1966). "Cénac M. Introduction théorique aux fonction de la psychanalyse en criminologie" (1950). In Écrits 1. Paris : Seuil.

Lacan, J. (1966). "Remarque sur le rapport de Daniel Lagache". In Écrits. Paris: Seuil.

Lacan, J. (1971). "Le savoir du psychanalyste". Conferência inédita proferida no hospital Saint Anne em 4 de novembro de 1971.

Lacan, J. (1950 [1966]). "Cénac M. Introduction théorique aux fonction de la psychanalyse en criminologie". In Ecrits 1. Paris: Seuil.

Lacan, J. (2001). "L'étourdit" (1973), Autres écrits. Paris: Seuil.

Lacan, J. (1974). Les non-dupes errent. Le Séminaire. Livre XXI, inédit du 11 Juin 1974.

Lacan, J. (1975a). Le Séminaire. Livre I, Les écrits techniques de Freud (Trabalho original publicado em 1953-1954). Paris : Seuil. 
Lacan, J. (1975b). Le Séminaire. Livre XX, Encore (Trabalho original publicado em 1972-1973). Paris: Seuil.

Lacan, J. (1985). Conférence de Genève sur "Le symptôme" du 4 Octobre 1975. Bloc-note de la psychanalyse.

Laurent, É. (1992). Discussion, in L'autisme et la psychanalyse. Presses Universitaires du Mirail.

Laurent, É. (2012). La bataille de l'autisme (De la clinique à la politique). Paris : Navarin.

Lefort, R.; Lefort, R. (1980). Naissance de l'Autre. Paris: Seuil.

Lefort, R.; Lefort, R. (1998). L'autisme, spécificité. In Fondation du champ freudien. Le symptômecharlatan. Paris: Seuil.

Lefort, R.; Lefort, R. (2003). La distinction de l'autisme. Paris: Seuil.

Lemay M. (2004). L'autisme aujourd'hui. Paris: Odile Jacob.

Luria, A.R. (1970). Une prodigieuse mémoire, Étude psycho-biographique. Delachaux et Niestlé, Neuchâtel.

Miller, J-A. (1975). "Théorie de lalangue", Ornicar?, Bulletin périodique du champ freudien.

Miller, J-A. (2005). "Pièces détachées", La Cause Freudienne, n61.

Mottron, L. (2004). Une outre intelligence. Editons Mardaga.

Mottron, L. (2016). L'intervention précoce pour enfants autistes. Paris : Mardaga.

Nazeer, K. (2006). Laissez entrer les idiots. Paris : Oh Editions.

Ouellette, A. (2011). Musique autiste. Paris :Triptyque.

Park, C. (1972). Histoire d'Elly (1967). Calmann-Lévy, Paris.

Peeters, T. (1996). L'autisme. De la compréhension à l'intervention. Paris : Dunod.

Richer, J. (1978). "L'absence partielle de transmission culturelle chez l'enfant autiste". In Rutter M.,

Schopler, E. (1991). L'autisme. Une réévaluation des concepts et du traitement, Paris: PUF.

Rothenberg, M. (1977/1979). Des enfants au regard de pierre). Paris: Seuil.

Saussure, F. (1972). Cours de linguistique générale. Paris: Payot.

Sellin, B. (1994). Une âme prisonnière (1993). Paris: Robert Laffont.

Sellin, B. (1998). La solitude du déserteur (1995). Paris: Robert Laffont.

Tammet, D. (2007). Je suis né un jour bleu (2006). Paris: Les Arènes.

Tammet, D. (2018). Conférence à I'Université Rennes 2; du 28 Mars 2018.

Tustin, F. (1977). Autisme et psychose de l'enfant (1972). Paris: Seuil.

Tustin, F. (1992). Autisme et protection (1990). Paris: Seuil.

Williams, D. (1992). Si on me touche, je n'existe plus. Paris: Robert Laffont.

Williams, D. (1994). Quelqu'un, quelque part. Paris : J'AI LU. 
Citacão/Citation: Maleval, J.C. (mai. 2018 a out. 2018). De la structure autistique. Revista aSEPHallus de Orientação Lacaniana, 13(26), 39-73. Disponível em www.isepol.com/asephallus. Doi: 10.17852/1809709x.2019v13n26p39-73.

Editor do artigo: Tania Coelho dos Santos.

Recebido/Received: 03/01/2019 / 01/03/2019.

Aceito/Accepted: 12/03/2019 / 03/12/2019.

Copyright: (C) 2019 Associação Núcleo Sephora de Pesquisa sobre o moderno e o contemporâneo. Este é um artigo de livre acesso, que permite uso irrestrito, distribuição e reprodução em qualquer meio, desde que o autor e a fonte sejam citados/This is an open-access article, which permites unrestricted use, distribution, and reproduction in any medium, provided the author and source are credited. 